\title{
From the library
}

\section{Remembrance of things past}

"Instead, as the late spring weather grew hotter, Francis' symptoms - especially the inflammation of his eyes-became increasingly serious. He couldn't stand sunlight and found it impossible to see by the glow of a lamp or candle at night. Imprisoned in darkness, his pain was so intense that he seldom rested or slept and if he did drop off was soon woken by the field mice that scampered all over him. Shocked to discover that he was giving way to self pity he concentrated harder on his prayers." (Adrian House. Francis of Assisi: A Revolutionary Life. Mahwah, New Jersey: Hidden Spring Press, 2000:268.)

\section{Sudden infant death syndrome and} serotonin

The aetiology of sudden infant death syndrome has yet to be determined. A recent study from Japan suggests that variations in 5-HT transporter genes may be related to sudden infant death syndrome. Serotonin $(5-\mathrm{HT})$ in the nervous system is a major factor in facilitating the brain centre for respiration. In this study there were significant differences in the genotype distribution and frequency of the 5-HT promoter gene in patients with sudden infant death syndrome. (Pediatrics 2001;107:690-2.)

Cholesterol levels and suicide

Recent reports have suggested that a link between low serum total cholesterol and the risk of death from suicide exists. In a study from Canada the mortality experience of the nutrition Canada survey revealed that patients in the lowest quartile of serum total cholesterol concentration had more than six times the risk of committing suicide than the highest. Why serum cholesterol levels are related to the risk of suicide is not known. (Epidemiology 2001;12:168-72.)

The risk of cardiac death and depression Depression may be a potential risk factor for subsequent cardiac death in patients with coronary artery disease. In a cohort study of 2847 men and women depression increased the risk for cardiac mortality in subjects with and without cardiac disease at baseline. The excess cardiac mortality risk was more than twice as high for major depression as it was for minor depression. (Arch Gen Psychiatry 2001; 58:221-7.)

\section{Oral therapy for CMV retinitis}

The FDA has approved valganciclovir for induction and maintenance therapy of cytomegalovirus retinitis in patients with AIDS. Valganciclovir is an orally administered prodrug of ganciclovir that provides improved bioavailability. The adverse effects of valganciclovir were comparable with those of approved ganciclovir formulations-namely, anaemia, neutropenia, and gastrointestinal symptoms. ( AMA 2001;285:2705.)

Suicide in doctors: women are at greater risk than men

A recent study investigating suicide in doctors in England and Wales demonstrated that there is an increased risk in female doctors compared with male doctors. In fact, male doctors had less risk than men in the general population. There were significant differences between specialties with anaesthesiologists, community health doctors, and psychiatrists having the highest risk of suicide. ( $\mathcal{F}$ Epidemiol Community Health 2001;55:296-300.)

\section{Coronary artery bypass surgery versus} stenting for treatment of multiple vessel disease

A study from Amsterdam has investigated the effectiveness of coronary artery bypass surgery versus stenting for patients with multiple coronary artery disease. At 1 year follow up there were no significant difference between the two groups in terms of the rate of death, stroke, or subsequent myocardial infarction. However, there was a $16.8 \%$ re-operation rate in the stenting group and only a 3.5\% re-operation rate in the surgical group. As the result of the much higher re-operation rate in the stenting group the cost effective difference between the two procedures was much les than would have been expected. ( $(F A M A$ 2001;285:2562.)

\section{Ginger relieves nausea and vomiting} during pregnancy

In a study from Thailand a randomised double masked placebo controlled trial has demonstrated that $1 \mathrm{~g}$ of ginger per day is effective for relieving the severity of nausea and vomiting associated with pregnancy. No adverse effects of ginger on pregnancy outcome were reported. (Obstet Gynecol 2001;97:577-82.)

How good is the medical information on the internet?

The internet is an increasingly important source of health related information for consumers. A recent survey estimated that more than 60 million US residents go on line each year to obtain health information. A recent study evaluated the health information on breast cancer, depression, obesity, and childhood asthma available through English and Spanish language search engines and websites. The study documented that accessing health information using existing search engines and simple terms is not efficient. This study also demonstrated that the coverage of key information on English and Spanish websites is poor and inconsistent, although the accuracy of information was generally of good quality. However, high reading levels are required to comprehend web based health information and a significant portion of the population can probably not comprehend it. (FAMA 2001;285:2612-21.)

\section{Green tea prevents ultraviolet injury to} the skin

In experimental animal models extracts from green tea have been shown to be remarkably effective at reducing the severity of ultraviolet damage. In a study from Alabama areas of skin of normal volunteers were treated with an extract of green tea or one of its constituents. On histological examinations, skin treated with green tea polyphenols showed fewer cells with burn damage and protection of the epidermis as well. Green tea extract also reduced the DNA damage that forms afte ultraviolet radiation. (F Am Acad Dermatol 2001;44:425-32.)
A new drug for leukaemia

A new drug ST1571 is a specific inhibitor of BCR-ABL tyrosine kinase. BCR-ABL is a constantly activated tyrosine kinase that causes chronic myeloid leukaemia. Thus, ST1571 is a new prototype for cancer therapy as a direct inhibitor of the chemical process required to initiate the malignant process. Adverse effects to the drug are minimal with the most common being nausea and diarrhoea. Complete haematological response was observed in 53 of 54 patients studied. (N Engl f Med 20001;344:103-37.)

\section{Penicillin allergies much less common} than reported

Clinicians frequently withhold antibiotics that contain penicillin based on patients' self reporting clinical history of an adverse effect of penicillin. In a recent study patients' self reported history of penicillin allergy was demonstrated to have a low accuracy. Only $10-20 \%$ of patients reporting a history of penicillin allergy are truly allergic to the drug when assessed by skin testing. Taking a detailed history of a patient's reaction to penicillin may allow clinicians to exclude penicillin allergy and thus allow the patient to receive penicillin. In those cases where penicillin allergy is still considered to be possible, skin testing may be a useful assessment tool. Virtually all patients with a negative skin test result can take penicillin without serious sequelae. ( $(A M A$ 2001;285:2504-5.)

\section{Remembrance of things past II}

By any standard, the visual brain is a remarkably efficient organ. It is capable of providing, within a fraction of a second, a visual image in which all the attributes of the scene-form, colour, motion, depth, and much else besides-are seen in precise special and temporal registration. It is an organ that is capable of recognising an object from a single view and of uniting many different views into a single object, without the apparent perceptual confusion that reigns in a Cubist painting such as Picasso's Man with a Violin, at least on first viewing. The most prominent victim of this efficiency has been the visual psychologist because it is this very efficiency that inhibited him, for a very long time, from inquiring into how the brain undertakes its remarkable task. (Seymir Zeki. Inner vision: an exploration of art and the brain. Oxford: Oxford Press, 1999:58.)

\section{Does long term oestrogen replacement} reduce the incidence of knee osteoarthritis?

Because osteoarthritis of the knee is more common in women than men the possible role of oestrogen in this joint disease has been suggested. In a new study using magnetic resonance imaging to measure the volume of knee cartilage, 81 post-menopausal women were examined; 42 had been oestrogen users whereas 39 age matched controls had not used oestrogen supplements. The results of this study suggest that the volume of knee cartilage in the group of women taking oestrogen supplements was approximately $8 \%$ more than in the controlled groups. Further longitudinal studies are under way. (Ann Rheum Dis 2001;60:332-6.) 